\title{
Translocated dogs from Nunavut and the spread of rabies
}

\author{
Curry PS ${ }^{1 *}$, Kostiuk $\mathrm{D}^{2}$, Werker $\mathrm{DH}^{1}$, Baikie $\mathrm{M}^{3}$, Ntiamoah $\mathrm{W}^{4}$, Atherton $\mathrm{F}^{5}$, Enns $\mathrm{A}^{6}$, Opondo $\mathrm{J}^{6}$, \\ Guirgis $\mathrm{H}^{7}$, Mema $\mathrm{S}^{8}$
}

\section{Abstract}

Background: Investigations of rabid animals that cross provincial/territorial boundaries are resource intensive and complex because of their multi-jurisdictional and multi-sectoral nature.

Objective: To describe the multi-jurisdictional responses to two unrelated rabid puppies originating from Nunavut.

Methods: A descriptive summary of the investigations following the identification of a rabid puppy in Alberta (August 2013) and another in Saskatchewan (December 2014).

Results: These investigations involved public health and agriculture authorities in five provinces/territories, as well as the Canadian Food Inspection Agency (CFIA). In Alberta, a puppy who became ill after being transported by air from Nunavut was euthanized and diagnosed with rabies (Arctic fox variant). Eighteen individuals were assessed for exposure to rabies; nine received rabies post-exposure prophylaxis (RPEP). An exposed household dog that tested negative was electively euthanized. In Nunavut, the rabid puppy's mother and litter mates were placed under quarantine. In Saskatchewan, another puppy became ill during transit by air from Nunavut. It was subsequently euthanized and diagnosed with rabies (Arctic fox variant). Two of three Saskatchewan individuals, including a veterinary technician, received RPEP. Two Nova Scotia residents were exposed to the puppy while in Nunavut and received RPEP. One household dog received booster vaccination, was quarantined for 45 days and remained asymptomatic. In Nunavut, the rabid puppy's mother and litter mates were not identified. In both cases, exposure to an Arctic fox was the probable source of rabies in the puppies.

Conclusion: Translocation of dogs from the north where Arctic fox rabies is endemic poses a risk to human and animal health and may negatively impact control of rabies in Canada. There is currently no national framework to prevent inter-jurisdictional movement of potentially rabid animals in Canada.

\section{Affiliations}

${ }^{1}$ Saskatchewan Ministry of Health, Population Health Branch, Regina, SK

${ }^{2}$ Alberta Agriculture and Forestry, Animal Health Branch, Edmonton, $A B$

${ }^{3}$ Nunavut Department of Health, Iqaluit, NU

${ }^{4}$ Nunavut Department of Health, Cambridge Bay, NU

${ }^{5}$ Nova Scotia Department of Health and Wellness, Halifax, NS

${ }^{6}$ Saskatoon Health Region, Saskatoon, SK

${ }^{7}$ Alberta Health Services, Environmental Public Health Services, Edmonton, $A B$

${ }^{8}$ Alberta Health Services, Calgary, $A B$ (currently with Interior Health Authority, BC)

\author{
*Correspondence: pcurry@ \\ health.gov.sk.ca
}

Suggested citation: Curry PS, Kostiuk D, Werker DH, Baikie M, Ntiamoah W, Atherton F, et al. Translocated dogs from Nunavut and the spread of rabies. Can Comm Dis Rep 2016;42:121-4. https://doi.org/10.14745/ ccdr.v42i06a02

\section{Introduction}

Between 2013 and 2014, two unrelated puppies, both less than one year old, were separately adopted and transported from Nunavut (NU) and diagnosed with rabies after arrival in their new home provinces. One puppy arrived in Calgary, Alberta $(A B)$, via Edmonton, $A B$, in August 2013 and the other arrived in rural Saskatchewan (SK), via Yellowknife, Northwest Territories (NT) and Edmonton, AB in December 2014. The investigations of both animal and human exposures collectively involved five provincial/territorial jurisdictions-NU, NT, AB, SK, as well as Nova Scotia (NS) - and the Canadian Food Inspection Agency (CFIA). Two residents of NS were exposed while working in NU. This outbreak report illustrates the complexity of these investigations due to their multidisciplinary nature and number of investigative partners, and highlights the challenges and gaps in national rabies control and prevention.

\section{Methods}

Two public health investigations were triggered by the diagnosis and notification of two confirmed rabid puppies that required trace backs and assessments of possible exposures of humans and animals to rabies. The purpose of these investigations was to prevent the spread of rabies to humans and animals. CFIA was responsible for animal investigations up to March 21, 2013, and was involved in the AB investigation. The SK Ministry of Agriculture was responsible for the animal investigation in SK. CFIA or territorial public health authorities were responsible for the animal investigations in NU and NT and the regional, provincial and territorial public health authorities were responsible for all human health investigations.

Regional/provincial/territorial public health and agriculture personnel who were directly involved in these two investigations 
reviewed their records and provided summaries that were compiled into this report by three authors (PSC, DK, DHW). A previously published report on the Alberta puppy (1) was updated by information obtained from $N U$ and a review of $A B$ records. The descriptive summaries were then reviewed for accuracy by the investigative team.

\section{Results}

\section{Alberta 2013}

In June of 2013, a Calgary resident working in a remote community in NU witnessed a family of puppies scavenging for food in the area where she worked. She wanted to bring one puppy home but was unable to as the puppies were too young to leave their mother. A few weeks later, a friend went up to the community and brought back a puppy for the Calgary resident. The puppy, a five-month old husky, was transported by air to Calgary from NU, via Edmonton.

The puppy was seen by a veterinary clinic in Calgary shortly after it arrived for first examination and vaccination on July 9, 2013. The owner reported that the puppy was doing well and acting like a normal puppy who had been rescued from a homeless situation.

The puppy was taken to the veterinary clinic for its second set of puppy vaccinations on August 8,2013 , and the decision was made to delay the rabies vaccine because the puppy needed a Bordetella vaccination. The puppy was adjusting well to its new life and the owner reported that it was happy, calm and quickly becoming housetrained.

The owner brought the puppy to the veterinary clinic four days later on August 12, 2013, because it had begun vomiting and attacking and biting the other dog in the household. The puppy had also bitten the owner's roommate on the back of the leg, but this was not a full epidermal thickness bite. It was howling intermittently and could not be soothed by the owner. The puppy was brought to the clinic in a carrier and was removed from the carrier using a rabies pole to muzzle and subsequently euthanize it. The veterinarian did not do a clinical exam on the puppy at this point as rabies was strongly suspected based on presenting clinical signs. The veterinarian consulted with the CFIA district veterinarian who explained that an unvaccinated dog suspected of having rabies would have to undergo a six-month quarantine period or be euthanized and tested for rabies. The owner elected to euthanize the puppy and test for rabies.

CFIA submitted the samples to the Rabies Laboratory in Lethbridge, AB on August 15, 2013. A positive fluorescent antibody test (FAT) was reported. On subsequent typing, the virus was found to be the Arctic fox variant of the rabies virus.

CFIA was also consulted regarding the management of the one-year-old dog in the household that had been bitten by the puppy. This dog had been vaccinated for rabies as a puppy but had not yet received the one-year booster vaccination and so was classified as a primary vaccinate animal. It was recommended that this dog either receive a booster vaccination immediately and then undergo a 45-day observation period, or be euthanized as per CFIA protocol. The owner elected euthanasia and the dog was tested for rabies; test results for this dog came back negative.

The CFIA Edmonton District office, which was responsible for reportable animal diseases in NU, contacted the owner of the puppies and placed a quarantine order on the remaining live puppies. Two of the puppies were destroyed for unrelated reasons shortly after the quarantine was issued. Both were healthy at the time of destruction. The mother and two remaining puppies remained healthy after the six-month quarantine ended. There was no report from the owner of an Arctic fox interacting with the dogs but they were housed outdoors and there had been an Arctic fox found to be positive in the community that winter.

Public health officials in $A B$ and NU assessed the exposures of 18 individuals who had contact with the puppy; nine were considered to have high-risk exposures and were given rabies post-exposure prophylaxis (RPEP). Four household members received RPEP, as well as four out of 12 acquaintances of the puppy's owners and one of the veterinarians that dealt with the puppy. One person received his/her last dose of rabies vaccine in NS.

\section{Saskatchewan 2014 to 2015}

A husky-like puppy, which was less than one year old, had wandered into a construction work camp in a remote NU community in early December 2014. It was apparently healthy and had been taken into the compound and nurtured by several of the workers at the camp. One of the workers decided to adopt the puppy and take it back to SK. On December 16, 2014, the puppy was transported on a flight from NU to Yellowknife, NT and then on another flight to Edmonton, AB. The owner then travelled by private vehicle to a rural community in the Saskatoon Health Region.

The puppy became progressively ill enroute with marked changes in its behaviour and bit a family member. The following day, the owner took the puppy to a local veterinary clinic. The veterinarian reported that the puppy's eyes were glazed, it was salivating heavily, was quite dysphoric, crying and throwing its head back and forth. As rabies was considered in the differential diagnosis, the puppy was euthanized and the head was sent to CFIA laboratory for testing. A public health investigation was initiated.

The veterinarian notified the Rabies Risk Assessment Veterinarian (RRAV) with SK Ministry of Agriculture about the possibility of the puppy being rabid. The RRAV notified public health in Saskatoon Health Region and the Ministry of Health and the Ministry of Agriculture of a possible rabid animal. The Saskatoon Health Region conducted a preliminary risk assessment of human exposures. Two co-workers of the owner had been exposed to the puppy at the camp in NU and had returned to NS. The bite to the family member resulted in puncture wounds of the arm, which received appropriate wound care at a physician's office. A veterinarian technician, who had previously received pre-exposure rabies vaccination, was cut with the knife used to 
decapitate the puppy's head after the knife had been immersed in a bleach solution.

CFIA reported that the FAT was positive for rabies on December 19. The RRAV notified public health officials and the Ministry of Agriculture of the positive test results. Subsequent typing confirmed Arctic fox variant rabies virus.

Both SK Ministry of Agriculture and SK Ministry of Health notified authorities in $A B$ about the rabid puppy on December 19. SK Ministry of Health also notified public health authorities in NU, NT and NS. As the owner of the puppy had already informed co-workers about the dog's illness, the co-workers had presented to public health in NS and had already been started on RPEP.

The family member bitten by the puppy was assessed as having been exposed to rabies and received RPEP. Since this person proceeded with pre-arranged travel plans, the final fourth dose was administered in Ireland (these arrangements were facilitated through the International Health Regulations National Focal Point in Ireland). Two other family members were assessed as not having been exposed to rabies. The veterinary technician received two booster vaccinations. Blood samples from all clinic staff were taken to determine rabies titres.

Public health in AB and NT investigated airport workers who might have come into contact with the puppy during transportation from NU. The puppy had been crated for the flights. There were no occupational exposures. AB public health authorities also confirmed that there were no exposures to humans during the puppy's road trip from Edmonton to its new home in SK.

Public health in NU investigated all individuals with potential exposure in the community. No one was offered RPEP. A public service announcement provided information to the community of the rabies situation and advice to avoid stray dogs and to report any dogs with unusual behaviour.

Since there are no veterinary or agricultural services in NU, public health authorities conducted the animal investigation. The rabid puppy's mother and litter mates in NU were not identified. However, on December 25, 2014, a young husky dog, foaming at the mouth, was found wandering around the same community. The dog was shot, sent for testing and was positive for Arctic fox variant rabies. Around the same time a fox was shot near the same community and also tested positive for Arctic fox variant rabies. There were no known human exposures to this animal.

The owner of the rabid puppy had another dog at home who had been exposed. This dog had been previously vaccinated against rabies but was not up-to-date. The SK Ministry of Agriculture required that the dog receive a rabies vaccine booster and be quarantined for a 45-day period by the owner. The dog remained well during the quarantine period and no further follow-up was required.

Investigations did not identify any other animals that were exposed to the rabid puppy.

In both events, human infections were prevented and there was no further spread of rabies to domestic animals, beyond the two puppies. Arctic fox variant rabies is endemic in many far northern communities. Dogs are exposed through contact with rabid Arctic foxes or other animals that have been infected by rabid Arctic foxes.

Approximately 25 public health and agriculture staff were directly involved in each of these investigations. This does not include resources that were required for human and animal health care services, laboratory testing or operational and public communications across the involved sectors.

\section{Discussion}

These two investigations constitute the second and third reports of translocation of rabid dogs within Canada. Similar incidents were reported in Quebec in 2012 (2). These cases highlight the risk that translocation of puppies from the north (where Arctic fox rabies is endemic) poses to human and animal health and its potential to negatively impact the control of rabies in Canada. These two investigations also demonstrate that the high risk of rabies in dogs from NU and other northern areas in Canada may not be appreciated by those living in other areas of Canada, where rabies is more commonly reported in skunks, bats and raccoons. In 2013 and 2014, 23\% and 27\% of the rabid animals identified from NU and NT were domestic dogs (3).

Unvaccinated dogs from NU and NT should be considered at high risk of having rabies, and humans identified through investigations to have significant exposures to these animals should be offered RPEP without waiting for test results. The relatively long (and variable) incubation period of rabies in dogs (on average three to 12 weeks) can lead to infected dogs appearing entirely healthy at the time of transportation out of the region $(4,5)$. Puppies represent a particular risk because they are desirable for adoption by humans at a time when they have not yet been vaccinated against rabies (6). In both of these investigations, domestic animals were also exposed, although none were infected. However, it should be noted that one of these dogs was euthanized possibly during the incubation period and may have developed rabies if it had lived. Furthermore, movement of these stray puppies to areas also inhabited by red foxes poses a risk of (re-)introduction of the Arctic fox rabies variant to wildlife in these areas.

When rabid animals cross jurisdictional boundaries, the complexity of investigations increases, which in turn may decrease the timeliness of interventions to prevent and control the spread of rabies. As CFIA no longer has responsibility for rabies risk assessment of animals and collection of animal specimens for rabies laboratory testing, each province and territory has developed its own rabies program resulting in a patchwork of programs without any clearly established operational protocols for coordinating trans-jurisdictional investigations. The absence of a standardized, national protocol may create delays in notification to other jurisdictions resulting in delayed identification and risk assessment of human or domestic animal exposures, actions that are critical to rabies prevention and control. The SK puppy investigation prompted the provincial Ministry of Health to develop a process map of investigations of animals at risk of having rabies, establish triggers for urgently reporting rabies events to the Ministry and implement a standard inter-jurisdictional referral form for animal exposure incidents (7). 
A proof of recent rabies vaccination is required for exotic or domestic animals imported into Canada from other countries (8). However, there is currently no vaccination requirement to prevent inter-jurisdictional movement of potentially rabid animals within Canada. Such a framework could help prevent situations such as these two animal cases of translocated rabies. The framework could include a combination of legislation/ regulations, policies, or guidance for domestic animals that travel across provincial/territorial boundaries to be vaccinated against rabies.

Collaboration and commitments from diverse stakeholders including the airline industry and transportation sector, the tourist industry, park authorities, trapping, hunting and outfitting associations and animal rescue groups, could inform the development of policies and communication materials supporting adherence to vaccinated animal movement. For example, animal rescue agencies could develop policies that rescued dogs be vaccinated and screened by a veterinarian before approving them for adoption. In addition, to protect both northern and southern residents, interventions are needed to improve vaccination coverage of domestic dogs in the north, such as paying lay vaccinators to conduct intensive vaccination clinics or drives, expanding the lay vaccinator program to other areas and supporting expanded vaccination clinics utilizing veterinarians from other jurisdictions. This would not only reduce the risk of translocation of rabies to southern regions, but would also bolster rabies prevention and control in northern Canada.

\section{Conclusion}

Inter-jurisdictional movement of humans and animals within Canada can easily result in the spread of rabies to areas where the disease is less common. A national program could incorporate the requirement for rabies vaccination for animals crossing provincial/territorial borders within Canada, best management practices and increasing awareness among Canadians of endemic rabies in the north. Without such a program, Canada may remain vulnerable to the spread of rabies across jurisdictions.

\section{Acknowledgments}

The authors would like to acknowledge the following individuals and organizations for their contributions to these investigations: Betty Althouse, Wendy Wilkins and Clarence Bischop (Rabies Risk Assessment Veterinarian), SK Ministry of Agriculture - Animal Health Unit; Lisa Haubrich, SK Ministry of Health; André Corriveau, Northwest Territories Department of Health and Social Services; Jack Muggaberg, Canadian Food Inspection Agency; Francis Nkemamin Ndemafia, Alberta Health Services; and the Ireland Health Service Executive Health Protection Surveillance Centre.

\section{Conflict of interest}

None.

\section{References}

1. Mema S, Friesen B, Desai S, Rock M, Mclntyre L. Rabies in a Cagary puppy adopted from the Arctic. Can J Public Health; 104(7)e510.

2. Abedelaziz N, Gravel G, Markowski F, Leblanc MA. Vigie - Interventions : Rage chez des chiots. Flash Vigie 2012; 7(2):Fév 2012. (Available only in French)

3. Canadian Food Inspection Agency (CFIA). Rabies in Canada. Ottawa ON: CFIA; 2016. http://www.inspection.gc.ca/ animals/terrestrial-animals/diseases/reportable/rabies/ rabies-in-canada/eng/1356156989919/1356157139999.

4. Greene CE, Rupprect CE. Rabies and other Lyssavirus infections. In: Greene CE, editor. Infectious diseases of the dog and cat. Philadelphia: WB Saunders; 2006. p. 167-183.

5. Fekadu M. Canine rabies. In: Baer GM editor. The natural history of rabies. Boca Ratton: CDC Press: 1991. p. 368-375.

6. White J, Wolfram KL, O'Connor B. Rabies in a 10-week old puppy. Can Vet. J 2007;48(9):931-934.

7. Saskatchewan Ministry of Health. Inter-jurisdictional referral following an animal exposure. In: Section 4: Vectorborne and zoonotic disease. Regina SK: Government of Saskatchewan; 2014. http://www.ehealthsask.ca/services/ manuals/Documents/cdc-section-4.pdf\#page $=26$.

8. Canadian Food Inspection Agency (CFIA). Import and travel requirements. Ottawa ON: CFIA; 2016. http://www. inspection.gc.ca/animals/terrestrial-animals/imports/policies/ live-animals/pets/eng/1326600389775/1326600500578CFIA. 